\title{
TMEM206 is a potential prognostic marker of hepatocellular carcinoma
}

\author{
LI ZHANG $^{1 *}$, SHI-YI LIU ${ }^{1 *}$, XIAO YANG ${ }^{2}$, YAN-QING WANG ${ }^{1}$ and YAN-XIANG CHENG ${ }^{1}$ \\ ${ }^{1}$ Department of Obstetrics and Gynecology, Renmin Hospital of Wuhan University, Wuhan, Hubei 430060; \\ ${ }^{2}$ Department of Obstetrics and Gynecology, Peking University People's Hospital, Beijing 100044, P.R. China
}

Received October 31, 2019; Accepted June 30, 2020

DOI: 10.3892/ol.2020.12035

\begin{abstract}
Transmembrane proteins are involved in the transportation of materials into and out of cells. The transmembrane protein (TMEM) family is a collection of poorly described transmembrane proteins that serve important roles in tumor development and progression. A number of TMEM proteins have been discovered. A newly discovered TMEM protein, TMEM206, transports ions across the membrane under physiological and pathological conditions, generating an acidic environment, which serves an important role in the microenvironment. However, the prognostic value and regulatory mechanisms of action of TMEM206 in tumors is unclear. The aim of the present study was to evaluate the prognostic value and regulation mechanisms of TMEM206 in tumors. Firstly, the expression of TMEM206 in tumors and normal tissues was assessed using the GEPIA and Oncomine databases and the results revealed that TMEM206 expression increased or decreased depending on the type of tumor. Subsequently, using the Human Protein Atlas and the Kaplan-Meier plotter, the findings of the present study revealed that TMEM206 is related to the prognosis of hepatocellular carcinoma. In order to explore the mechanism of TMEM206 in promoting tumor progression, GEO and cBioPortal were used to determine genes that may be co-expressed with TMEM206. MetaScape was used to identify the signaling pathways that TMEM206 may participate in. Finally, miRWalk, miRDB and TargetScan were used to identify miRNAs that may regulate the expression of TMEM206 and the findings revealed that 2 miRNA (hsa-miR-325 and hsa-miR-510-5p) were involved. In conclusion, upregulation of TMEM206 is associated with poor prognosis in patients with hepatocellular carcinoma.
\end{abstract}

Correspondence to: Dr Yan-Xiang Cheng, Department of Obstetrics and Gynecology, Renmin Hospital of Wuhan University, 238 Jiefang Road, Wuhan, Hubei 430060, P.R. China

E-mail: doctornancy@qq.com

*Contributed equally

Key words: transmembrane protein 206, tumor microenvironment, hepatocellular carcinoma

\section{Introduction}

Hepatocellular carcinoma (HCC) is the sixth most common malignant tumor globally and $>700,000$ people die from this disease every year (1). Patients with HCC do not present with obvious symptoms until advanced stages of the disease, making treatment difficult and ineffective (2). Hence, it is vital to identify some potential biomarkers for the diagnosis or prognosis of HCC.

The extracellular environment of solid tumors is acidic, with a $\mathrm{pH}$ between 6.5 and 6.9 , whereas normal tissues is alkaline, with a pH between 7.2 and 7.5 (3). Tissue acidosis can result in tumor progression (4). Therefore, interventions targeting the acidic microenvironment of tumors may provide new therapeutic opportunities. However, the mechanism of action behind the generation of the acidic microenvironment in tumorigenesis is still in the initial stage, with further research urgently needed.

In order to maintain the stability of the $\mathrm{pH}$, tumor cells transport acidic substances to the outside or transport extracellular basic substances into the cells through a series of transport proteins, such as the $\mathrm{H}^{+}$-ATPase and the $\mathrm{Na}^{+} / \mathrm{H}^{+}$exchanger 1 (5). The transmembrane protein (TMEM) family contains proteins that span the entire width of the lipid bilayer and are permanently immobilized (6). A number of TMEMs act as channels, allowing specific substances to be transported between the intracellular and extracellular environment (7). However, the functions of TMEMs are unclear, and investigations into their functions are urgently required.

TMEMs are present in various cell types and are involved in a number of important physiological processes. For example, TMEM16A acts as a calcium-activated chloride channel $(8,9)$, whereas TMEM132A may be involved in brain development during the embryonic stages (10). Furthermore, studies have confirmed that TMEMs may serve an important role in tumor growth and development $(11,12)$. For instance, TMEM88 binds to disheveled proteins and promotes the invasion and metastasis of non-small cell lung cancer by activating the p38/glycogen synthase kinase $3 \beta /$ Snail pathway (12).

Recently, Yang et al demonstrated that one type of $\mathrm{CI}^{-}$ channels was activated by an acidic extracellular $\mathrm{pH}$, and it participated in the proton-activated $\mathrm{CI}^{-}$(PAC) currents; this 
channel protein was confirmed to be TMEM206, also termed PAC or pacc1 (13). However, little is known about TMEM206. Based on the results of the aforementioned study, it may be speculated that TMEM206 also serve a fundamental role in tumorigenesis and progression. To improve the understanding of TMEM206, the present study aimed to examine the expression profiling and prognostic values of TMEM206 in human cancers using multiple public databases, and potential co-expression genes that may be associated with the dysregulation of TMEM206 in hepatocellular carcinoma (HCC) were explored, which may be beneficial for the further study of TMEM206.

\section{Materials and methods}

Expression profiling of TMEM206 in human cancers. Gene expression profiling interactive analysis (GEPIA, http://gepia.cancer-pku.cn) and Oncomine (http://www. oncomine.org) databases were used to explore the potential features of TMEM206 in human tumors and normal tissues. GEPIA software is a web tool that analyzes the RNA expression data from The Cancer Genome Atlas (TCGA) and Genotype-Tissue Expression (GTEx) projects (https://www. cancer.gov/) (14). The Oncomine database is a web server used for bioinformatics services containing 715 datasets that provides a large-scale, high quality and consistent analytical method for gene expression profile analysis (15).

Pan-cancer survival analysis. The Kaplan-Meier plotter (http://kmplot.com//analysis) (16) was used to assess the prognostic roles of TMEM206 in 10,461 cancer samples. The forest plot was constructed using the $\mathrm{R}$ programming language (17). The Human Protein Atlas (HPA) dataset (https://www.proteinatlas.org/) $(18,19)$ is based on TCGA and was used to analyze the association between TMEM206 RNA and protein expression and overall survival rate of patients with HCC. Additionally, the UCSC Xena browser (http://xena. ucsc.edu) (20) was used to evaluate the association between TMEM206 mRNA expression levels and the prognosis of patients with HCC. Due to the crossing of survival curves, Simon's two-stage test was used rather than the log-rank test $(21,22)$.

Potential transcription regulatory mechanisms of TMEM206. The cBioPortal for Cancer Genomics (http://www.cbioportal. org) was used as a web resource to analyze and visualize the genomics data. The cBioPortal was also used to obtain the potential co-expression genes of TMEM206 in HCC (ISpearman's correlation coefficient $1>0.4$ ) (23). Additionally, the potential co-expression genes of TMEM206 were extracted from Gene Expression Omnibus (GEO; https://www.ncbi.nlm. nih.gov/geo/) datasets GSE36376 and GSE76427, separately, which have previously been used to analyze gene expression for tumor and adjacent non-tumor tissues in $\operatorname{HCC}(24,25)$. To identify the genes co-expressed with TMEM206, a Spearman's correlation test was used by the R platform (ISpearman's correlation coefficientl>0.4) (26). Finally, using online Venn software (http://bioinformatics.psb.ugent.be/webtools/Venn/), the intersection of these gene lists was determined as the co-expression genes of TMEM206 in HCC.
The miRWalk (27,28), miRDB (29) and TargetScan (30) databases were used to identify potential interacting microRNAs (miRNAs/miRs) (TargetScan, context++ score percentile $>80$; miRDB, score $>80$ and miRWalk: Score $>0.8$ ). The shared miRNAs among the three sets of prediction results were regarded as potential miRNAs that target TMEM206 mRNA.

Functional annotation of a co-expression gene network of TMEM206. Kyoto Encyclopedia of Genes and Genomes (KEGG) $(31)$ and gene ontology (GO) $(32,33)$ are commonly used for the functional annotations of genes. MetaScape (https://metascape.org/gp/index.html\#/main/step1) is an integrated analytics platform that integrates multiple annotation datasets. The functional enrichment analysis was performed using these platforms to analyze the function of TMEM206 co-expression genes (34).

\section{Results}

Expression of TMEM206 in cancer. The distribution of TMEM206 expression in various tissues of the human body based on TCGA and GTEx projects was obtained from the GEPIA website (Fig. 1A). TMEM206 was observed to be abundant in human tissues, especially in the brain and blood (Fig. 1B). Subsequently, the differential expression of TMEM206 in human tumor types and normal control tissues was investigated using the Oncomine database; the results demonstrated that 30 out of 150 tumors exhibited TMEM206 upregulation, and eight exhibited downregulation compared with that in normal tissues (Fig. 1C). Although, TMEM206 was abundant in normal and malignant tissues, significant increases in TMEM206 expression level were observed in colorectal, breast and liver cancer and lymphoma, and decreased levels were observed in kidney cancer compared with those in the corresponding normal tissues.

Pan-cancer survival analysis of TMEM206 mRNA expression suggests a prognostic role of TMEM206 in HCC. The Kaplan-Meier plotter datasets were used for pan-cancer survival analysis. The results demonstrated that TMEM206 exhibited notable prognostic significance in 5 of the 21 analyzed types of tumors (Table SI). Heterogeneity among various types of cancer was observed (Fig. 2). A higher expression level of TMEM206 was associated with a poor prognosis of head and neck squamous cell carcinoma, renal papillary cell carcinoma, HCC and sarcoma, while a lower expression level of TMEM206 was associated with a poor prognosis of thymoma (Fig. 2). Subsequently using the HPA dataset, which is based on experimental data, it was found that high protein expression level of TMEM206 was related to a poor survival rate of HCC (Fig. 3). Therefore, the present study focused on TMEM206 in HCC for the subsequent investigation.

The UCSC Xena browser was used to evaluate the association between the DNA variants and upregulation of TMEM206 in HCC. Expression was colored red to blue for high to low expression (Fig. 4A). Survival analysis using the Simon's two-stage test (low expression, $\mathrm{n}=212$ and high expression, $\mathrm{n}=206$, respectively) demonstrated that high expression of 
A

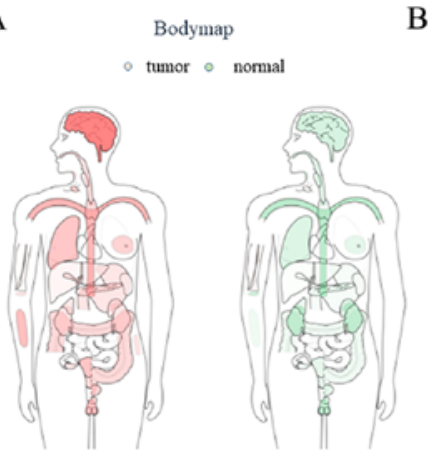

B

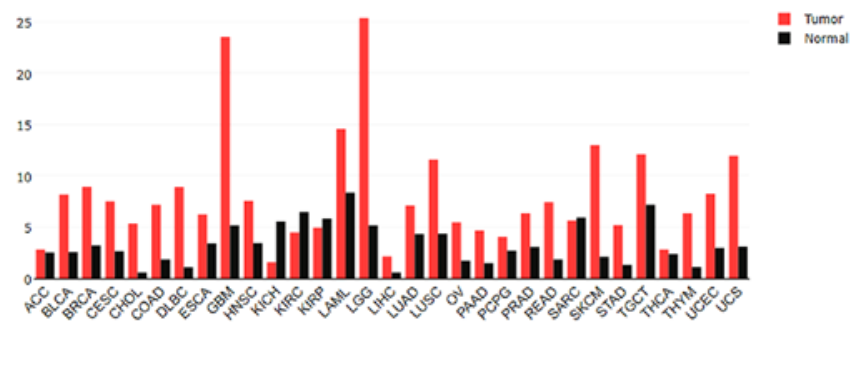

C

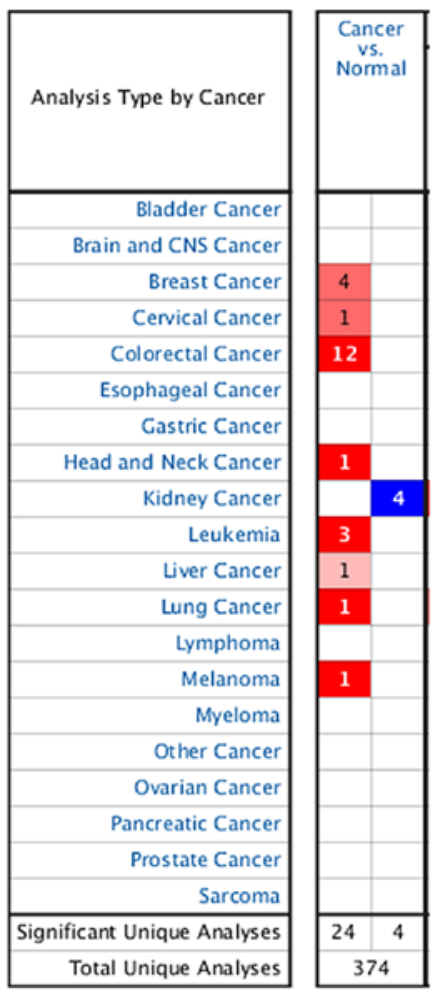

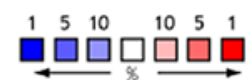

Figure 1. Pan-cancer expression profiling analysis of TMEM206. (A) Body map of TMEM206 in GEPIA; a darker color indicates a higher gene expression level. Red represents the expression of TMEM206 in tumor specimens, and green represents that in normal specimens. (B) Bar chart of the number of studies in which TMEM206 expression was significantly different in normal tissues compared with tumor tissues. (C) Boxplot of the pan-cancer expression profiling of TMEM206 in human cancers. Cell color is determined by the best gene rank percentile for the analyses within the cell; an analysis may be counted in more than one type of cancer. The color refers to the tumor (red) or normal tissue (blue). GEPIA, Gene expression profiling interactive analysis; TMEM, transmembrane protein.

TMEM206 mRNA was an unfavorable prognostic marker for patients with HCC (Fig. 4B). However, the methylation status and copy number variant of TMEM206 DNA were not associated with survival in HCC (Fig. 4C and D). As there was no significant difference, the Simon's two-stage test was not used inspite of the crossover of survival curves.

Functional annotation of the TMEM206 co-expression gene network. Co-expression gene analysis was used to further investigate the possible functions of TMEM206 in HCC. Data were acquired from cBioPortal and the GEO datasets GSE36376 and GSE76427 (ISpearman's correlation coefficient $|>0.4|$ ). The intersections of the three gene lists in the
Venn diagram were considered to be potential co-expression genes for TMEM206 (Fig. 5A; Table SII). MetaScape was used to analyze functional gene enrichment; the most significantly enriched GO term was the 'U2-type spliceosomal complex', which suggested that may regulate the expression of TMEM206 and the co-expression genes associated with it (Fig. 5B).

In addition, the miRWalk, miRDB and TargetScan databases were used to identify the potential miRNAs involved in the regulation of TMEM206. With strict screening criteria, two microRNAs, hsa-miR-325 and hsa-miR-510-5p (also termed has-miR-510) that may bind to TMEM206 mRNA were identified (Fig. 6A). Kaplan-Meier survival analysis 


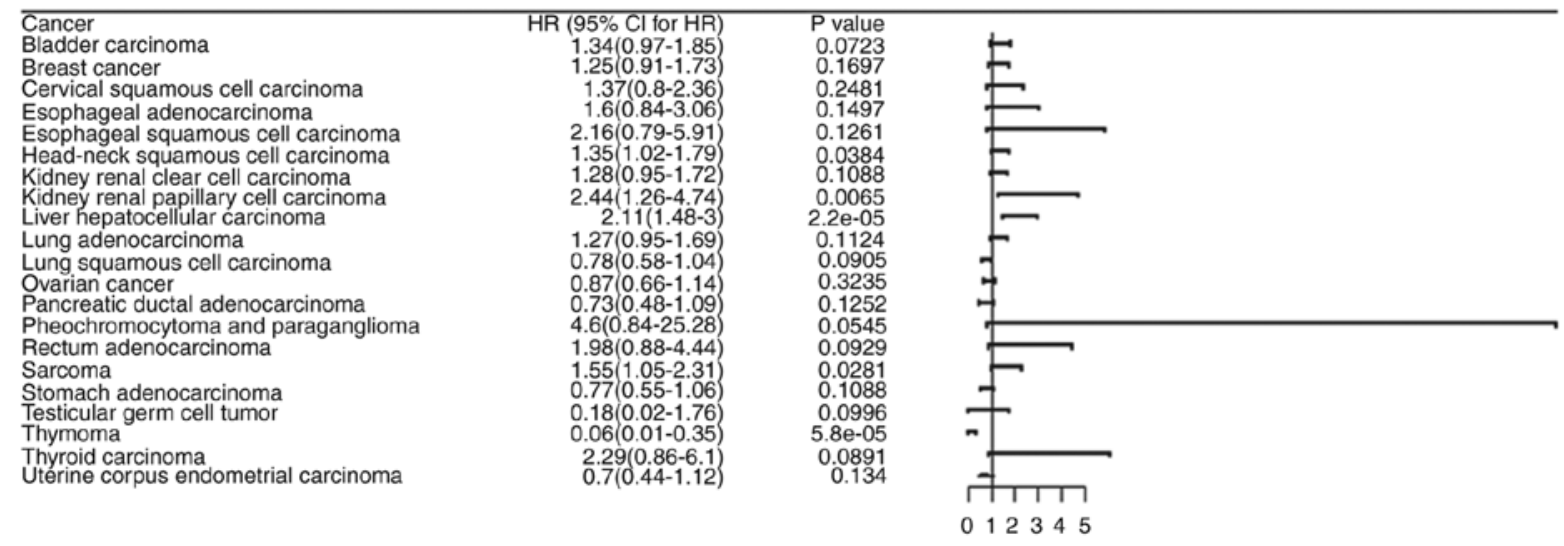

Figure 2. Forest plot of the pan-cancer survival analysis.

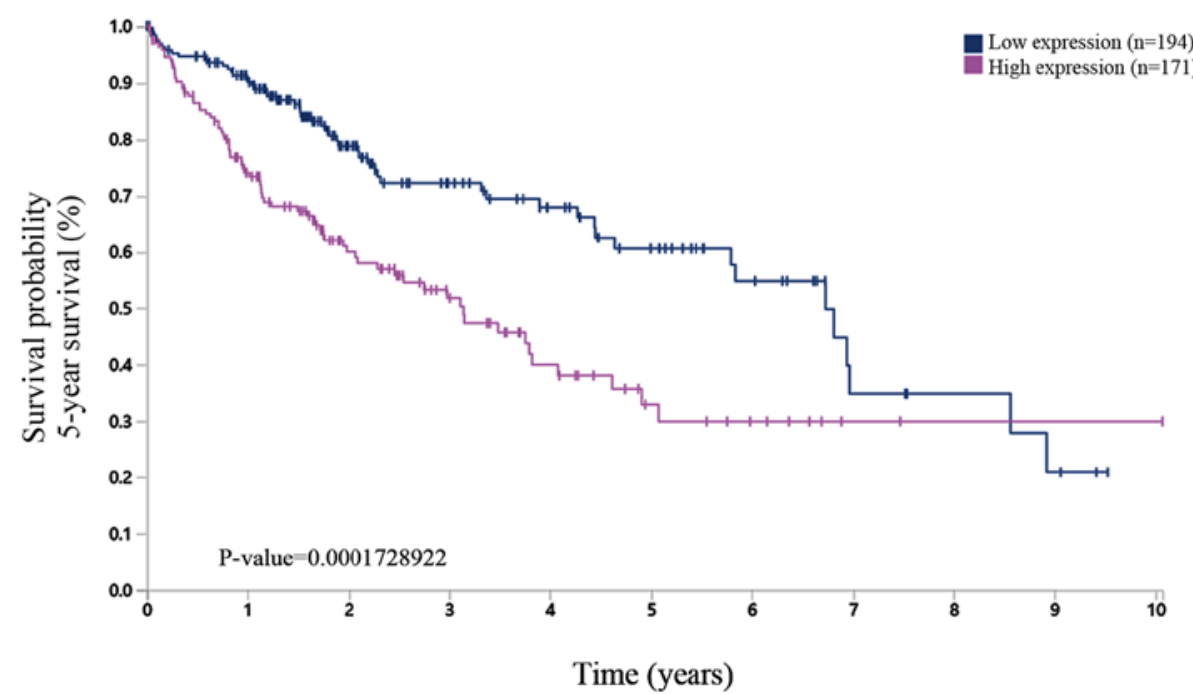

Figure 3. The association between the survival rate (\%) and transmembrane protein 206 protein expression levels. Data were analyzed using a Simon's two-stage test.

demonstrated that hsa-miR-325 and hsa-miR-510-5p were associated with the prognosis of patients with HCC. High expression levels of hsa-miR-325 and hsa-miR-510-5p were favorable for overall survival $\left(\mathrm{P}=2.3 \times 10^{-10}\right.$ and $\mathrm{P}=7.2 \times 10^{-10}$, respectively; Fig. $6 \mathrm{~B}$ and $\mathrm{C}$ ), suggesting that hsa-miR-325 and hsa-miR-510-5p expression levels were negatively associated with TMEM206 in HCC.

\section{Discussion}

Numerous studies have demonstrated that TMEM expression is up- or downregulated in tumor tissues compared with that in adjacent healthy or benign tissues $(35,36)$. For example, TMEM97 acts as prognostic biomarker for non-small cell lung cancer (35). Furthermore, a previous study has reported that TMEM proteins are involved in tumorigenesis and cancer development, such as TMEM45A, which participates in the proliferation and invasion of ovarian cancer cells (37). Therefore, an improved understanding of the TMEM family may help identify their functions in various types of cancer and improve therapeutic strategies.
TMEM206 is a newly identified transmembrane protein, Yang et al (13) performed unbiased RNA interference screening and demonstrated that TMEM206 was essential for the widely observed PAC currents $\left(\mathrm{I}_{\mathrm{Cl}, \mathrm{H}}\right)$, which are involved in acid-sensing ion channels. Little is known about the role of TMEM206 in tumors. Zhao et al (38) reported that TMEM206 mRNA and protein expression levels were higher in colorectal cancer (CRC) tissues compared with adjacent non-tumor tissues, overexpression of TMEM206 promoted the proliferation and invasion of CRC cells and positively regulated the levels of phospho-AKT and its downstream signaling pathway components, suggesting that TMEM206 promoted the development and progression of CRC by enhancing the interactions between the AKT and ERK signaling pathways.

A previous study has reported that the TMEM family is widely expressed in mammalian cells (39), which is in accordance with the present study. In the present study, the results of the expression profiling analysis demonstrated that TMEM206 expression was significantly increased in colorectal cancer, breast cancer, lymphoma and liver cancer, and reduced in the kidney cancer datasets. In terms of a prognostic role for 
A

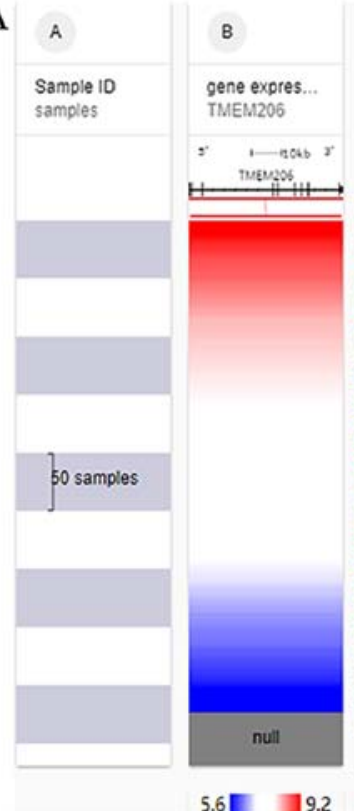

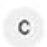
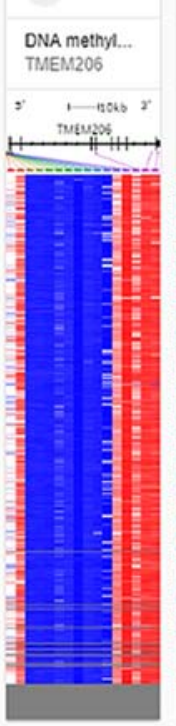

0.0 T 1.0

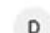

copy number... TMEM206

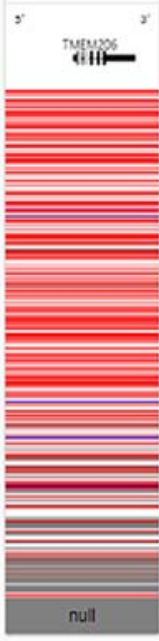

$-0.63[] 0.63$
B

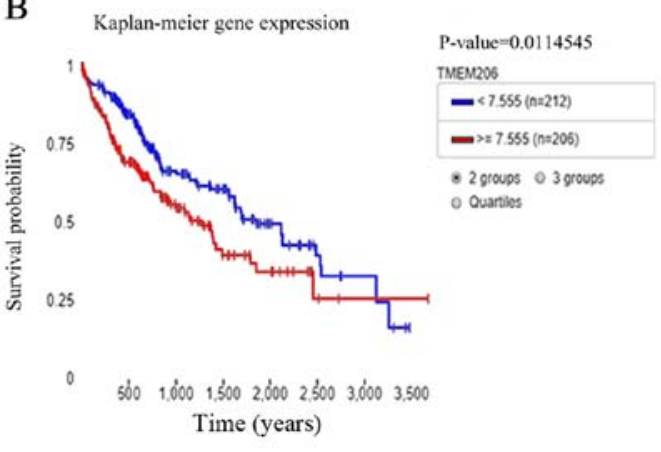

D

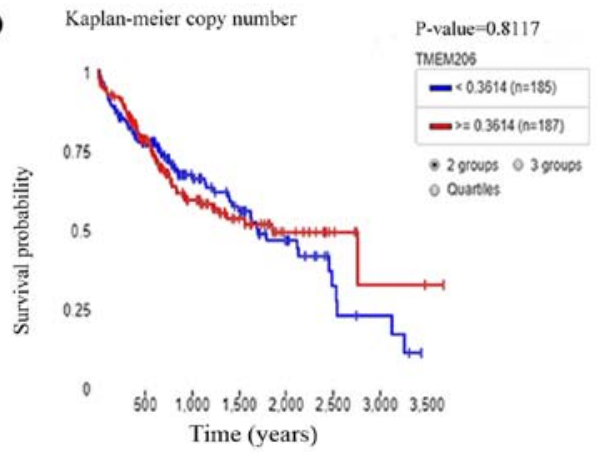

Figure 4. Association between the expression level, methylation status and copy number variant of TMEM206 and the prognosis of patients with HCC. (A) The heat map of the expression level, methylation status and copy number variant of TMEM206. (B-D) Kaplan Meier plots of the association between the (B) expression level, (C) methylation status or (D) copy number variant of TMEM206 and the prognosis of patients with HCC were analyzed by a two-stage test. HCC, hepatocellular carcinoma; TMEM, transmembrane protein.
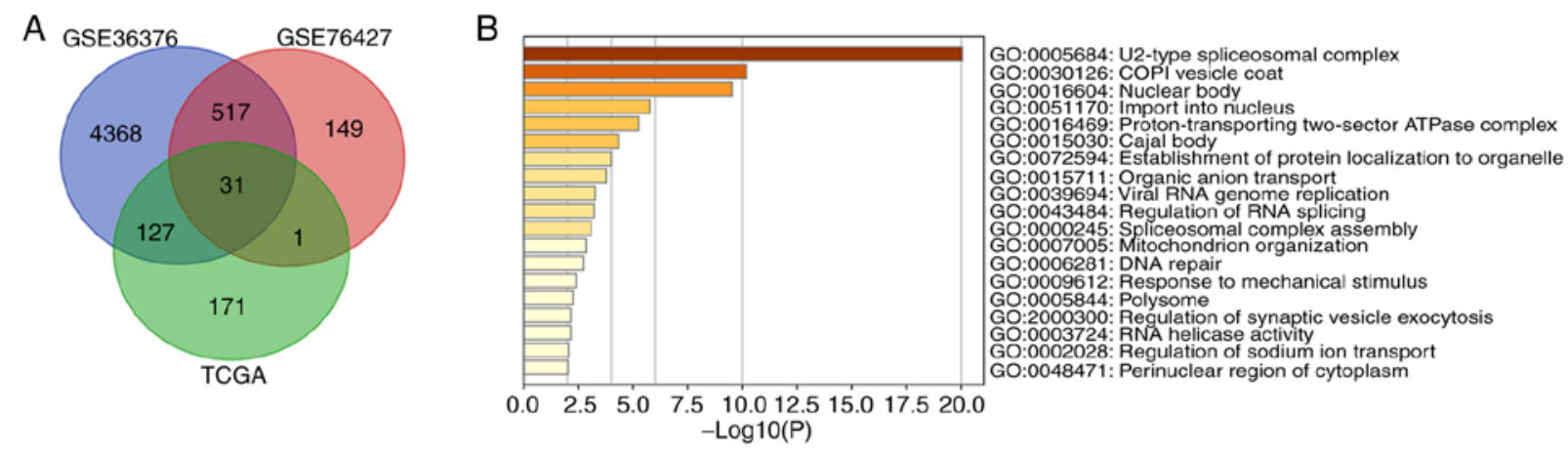

Figure 5. Venn plot and Metascape enrichment results of co-expression genes for TMEM206. (A) The intersection of gene lists (TCGA, GSE76427 and GSE36376 HCC) identified as co-expressed genes for TMEM206. (B) The enriched terms of the co-expressed genes for TMEM206. TCGA, The Cancer Genome Atlas; HCC, hepatocellular carcinoma; TMEM, transmembrane protein; COPI, coat protein complex I.

TMEM206, the present study demonstrated that TMEM206 was a significant prognostic factor for certain types of tumor and served either protective or unfavorable roles depending on the tumor type. These contrary results make the role of TMEM206 dubious.
Further analysis of data from patients with HCC demonstrated that the upregulation of TMEM206 was an unfavorable prognostic factor for HCC. TMEM206 was associated with the overall survival probability and 5-year survival rate of patients with liver cancer in The Kaplan Meier plotter and HPA. In 


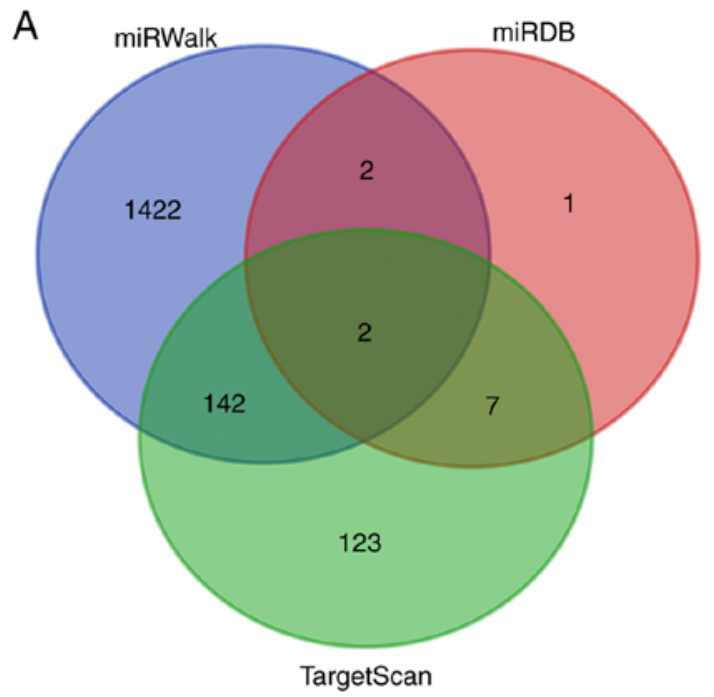

B

운

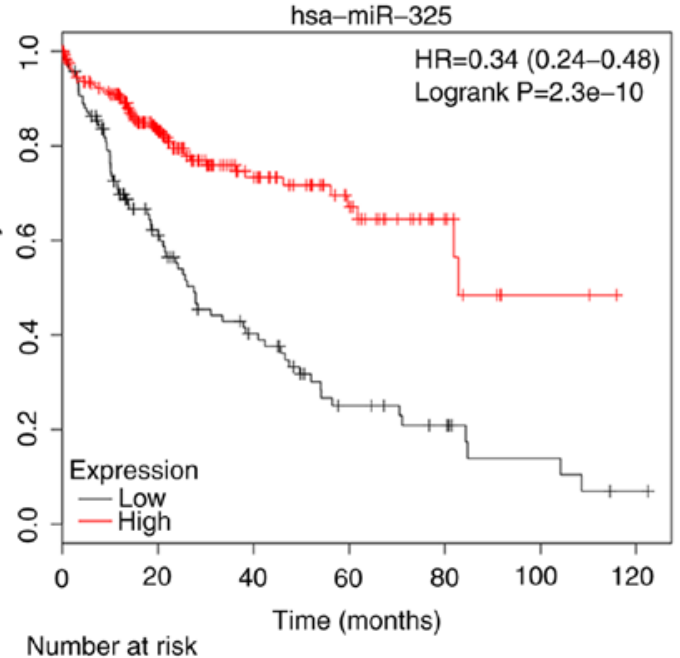

Now 124 at risk

W 12455

High $247 \quad 130$
C

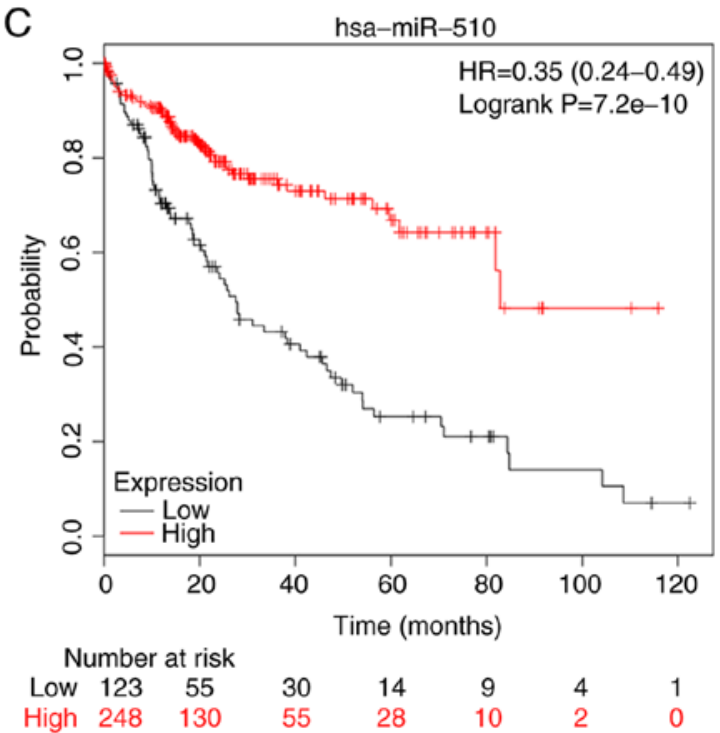

Figure 6. Potential microRNAs involved in the regulation of TMEM206. (A) Potential microRNAs involved in the regulation of TMEM206.(B and C) Association between $(\mathrm{B})$ hsa-miR-325 and $(\mathrm{C})$ hsa-miR-510-5p and overall survival time in patients with hepatocellular carcinoma $\left(\mathrm{P}=2.3 \times 10^{-10}, \mathrm{P}=7.2 \times 10^{-10} ;\right.$ respectively). miR, microRNA; TMEM, transmembrane protein.

addition, in the present study, the UCSC Xena browser was used to evaluate the association between the DNA variants and upregulation of TMEM206 in HCC. However, no significant associations were observed between the copy number variant or the extent of methylation of TMEM206 and the prognosis of patients with HCC. GEO and cBioPortal were used to determine genes co-expressed with TMEM206 and 31 genes were discovered. Using MetaScape, the signalling pathways that co-expression genes of TMEM206 may participate in were identified and the term U2-type spliceosomal complex was most significant. Finally, the present study identified two potential miRNAs, hsa-miR-325 and hsa-miR-510-5p, that may target TMEM206.

Hsa-miR-325 and hsa-miR-510-5p participate in tumor development (40-43). Hsa-miR-325 has been identified as a potential biomarker in human bladder cancer and low expression of hsa-miR-325 was associated with poor overall survival in patients with bladder cancer (40). In addition, downregulation of hsa-miR-325 has been demonstrated to promote the progression of non-small cell lung cancer and HCC $(41,42)$. Chen et al $(43)$ found that hsa-miR-510-5p acted as a tumor suppressor in renal cell carcinoma by reducing cell proliferation, migration and inducing apoptosis.

In conclusion, the results of the present study demonstrated that upregulation of TMEM206 is a potential prognostic indicator for HCC. However, studies into the role of TMEM206 are still at the early stage, and further research is required to clarify its role in HCC and other types of tumors.

\section{Acknowledgements}

Not applicable.

\section{Funding}

This study was supported by the National Natural Science Foundation of China (grant no. 81860276). 


\section{Availability of data and materials}

The datasets used/and or analyzed during the current study are available from the corresponding author on reasonable request.

\section{Authors' contributions}

LZ, SYL, XY, YQW and YXC contributed toward data analysis, drafting and revising the manuscript. All authors read and approved the final manuscript.

\section{Ethics approval and consent to participate}

Not applicable.

\section{Patient consent for publication}

Not applicable.

\section{Competing interests}

The authors declare that they have no competing interests.

\section{References}

1. Zeng Z, Dong J, Li Y, Dong Z, Liu Z, Huang J, Wang Y, Zhen $\mathrm{Y}$ and $\mathrm{Lu} \mathrm{Y}$ : The expression level and diagnostic value of microRNA-22 in HCC patients. Artif Cells Nanomed Biotechnol 48: 683-686, 2020.

2. Heimbach JK, Kulik LM, Finn RS, Sirlin CB, Abecassis MM, Roberts LR, Zhu AX, Murad MH and Marrero JA: AASLD guidelines for the treatment of hepatocellular carcinoma. Hepatology 67: 358-380, 2018.

3. Griffiths JR: Are cancer cells acidic? Br J Cancer 64: 425-427, 1991.

4. Alfarouk KO, Ahmed SB, Ahmed A, Elliott RL, Ibrahim ME, Ali HS, Wales CC, Nourwali I, Aljarbou AN, Bashir AH, et al: The interplay of dysregulated $\mathrm{pH}$ and electrolyte imbalance in cancer. Cancers (Basel) 7: 898, 2020.

5. White SH: Biophysical dissection of membrane proteins. Nature 459: 344-346, 2009.

6. Jung SJ, Jung Y and Kim H: Proper insertion and topogenesis of membrane proteins in the ER depend on sec63. Biochim Biophys Acta Gen Subj 1863: 1371-1380, 2019.

7. Marx S, Dal Maso T, Chen JW, Bury M, Wouters J, Michiels C and Le Calvé B: Transmembrane (TMEM) protein family members: Poorly characterized even if essential for the metastatic process. Semin Cancer Biol 60: 96-106, 2020.

8. Hartzell HC, Yu K, Xiao Q, Chien LT and Qu Z Anoctamin/TMEM16 family members are $\mathrm{Ca}^{2+}$-activated Cl-channels. J Physiol 587: 2127-2139, 2009.

9. Yang YD, Cho H, Koo JY, Tak MH, Cho Y, Shim WS, Park SP Lee J, Lee B, Kim BM, et al: TMEM16A confers receptor-activated calcium-dependent chloride conductance. Nature 455 : 1210-1215, 2008.

10. Oh-hashi K, Imai K, Koga H, Hirata Y and Kiuchi K: Knockdown of transmembrane protein $132 \mathrm{~A}$ by RNA interference facilitates serum starvation-induced cell death in neuro2a cells. Mol Cell Biochem 342: 117-123, 2010.

11. Zhao Y, Song K, Zhang Y, Xu H, Zhang X, Wang L, Fan C, Jiang $\mathrm{G}$ and Wang E: TMEM17 promotes malignant progression of breast cancer via AKT/GSK3 $\beta$ signaling. Cancer Manag Res 10: 2419-2428, 2018.

12. Zhang X, Yu X, Jiang G, Miao Y, Wang L, Zhang Y, Liu Y, Fan C, Lin X, Dong Q, et al: Cytosolic TMEM88 promotes invasion and metastasis in lung cancer cells by binding DVLS Cancer Res 75: 4527-4537, 2015.

13. Yang J, Chen J, Del Carmen Vitery M, Osei-Owusu J, Chu J, $\mathrm{Yu}$ H, Sun S and Qiu Z: PAC, an evolutionarily conserved membrane protein, is a proton-activated chloride channel. Science 364: 395-399, 2019.
14. Tang Z, Li C, Kang B, Gao G, Li C and Zhang Z: GEPIA: A web server for cancer and normal gene expression profiling and interactive analyses. Nucleic Acids Res 45: W98-W102, 2017.

15. Rhodes DR, Kalyana-Sundaram S, Mahavisno V, Varambally R, Yu J, Briggs BB, Barrette TR, Anstet MJ, Kincead-Beal C, Kulkarni P, et al: Oncomine 3.0: Genes, pathways, and networks in a collection of 18,000 cancer gene expression profiles. Neoplasia 9: 166-180, 2007.

16. Menyhárt O, Nagy Á and Győrffy B: Determining consistent prognostic biomarkers of overall survival and vascular invasion in hepatocellular carcinoma. R Soc Open Sci 5: 181006, 2018.

17. Gentleman RC, Carey VJ, Bates DM, Bolstad B, Dettling M, Dudoit S, Ellis B, Gautier L, Ge Y, Gentry J, et al: Bioconductor: Open software development for computational biology and bioinformatics. Genome Biol 5: R80, 2004

18. Uhlen M, Fagerberg L, Hallström BM, Lindskog C, Oksvold P, Mardinoglu A, Sivertsson Å, Kampf C, Sjöstedt E, Asplund A, et al: Proteomics. Tissue-based map of the human proteome. Science 347: 1260419, 2015.

19. Thul PJ, Akkesson L, Wiking M, Mahdessian D, Geladaki A, Ait Blal H, Alm T, Asplund A, Björk L, Breckels LM, et al: A subcellular map of the human proteome. Science 356: 6340, 2017.

20. Goldman M, Craft B, Hastie M, Repečka K, Kamath A, McDade F, Rogers D, Brooks AN, Zhu J and Haussler D: The UCSC Xena platform for public and private cancer genomics data visualization and interpretation. bioRxiv 326470, 2019.

21. Li H, Han D, Hou Y, Chen H and Chen Z: Statistical inference methods for two crossing survival curves: A comparison of methods. PLoS One 10: e0116774, 2015.

22. Qiu P and Sheng J: A two-stage procedure for comparing hazard rate functions. J Royal Statistical Soc 70: 191-208. 2008.

23. Cerami E, Gao J, Dogrusoz U, Gross BE, Sumer SO, Aksoy BA, Jacobsen A, Byrne CJ, Heuer ML, Larsson E, et al: The cBio cancer genomics portal: An open platform for exploring multidimensional cancer genomics data. Cancer Discov 2: 401-404, 2012.

24. Lim HY, Sohn I, Deng S, Lee J, Jung SH, Mao M, Xu J, Wang K, Shi S, Joh JW, et al: Prediction of disease-free survival in hepatocellular carcinoma by gene expression profiling. Ann Surg Oncol 20: 3747-3753, 2013

25. Grinchuk OV, Yenamandra SP, Iyer R, Singh M, Lee HK, Lim KH, Chow PK and Kuznetsov VA: Tumor-adjacent tissue co-expression profile analysis reveals pro-oncogenic ribosomal gene signature for prognosis of resectable hepatocellular carcinoma. Mol Oncol 12: 89-113, 2018.

26. Tang S, Jing H, Huang Z, Huang T, Lin S, Liao M and Zhou J: Identification of key candidate genes in neuropathic pain by integrated bioinformatic analysis. J Cell Biochem 121: 1635-1648, 2020.

27. Sticht C, De La Torre C, Parveen A and Gretz N: MiRWalk: An online resource for prediction of microRNA binding sites. PLos One 13: e0206239, 2018.

28. Du A, Zhao S, Wan L, Liu T, Peng Z, Zhou Z, Liao Z and Fang H: MicroRNA expression profile of human periodontal ligament cells under the influence of Porphyromonas gingivalis LPS. J Cell Mol Med 20: 1329-1338, 2016.

29. Liu W and Wang $X$ : Prediction of functional microRNA targets by integrative modeling of microRNA binding and target expression data. Genome Biol 20: 18, 2019.

30. Agarwal V, Bell GW, Nam JW and Bartel DP: Predicting effective microRNA target sites in mammalian mRNAs. ELife 4: e05005, 2015.

31. Kanehisa M, Furumichi M, Tanabe M, Sato $Y$ and Morishima K: KEGG: New perspectives on genomes, pathways, diseases and drugs. Nucleic Acids Res 45: D353-D361, 2017.

32. Ashburner M, Ball CA, Blake JA, Botstein D, Butler H, Cherry JM, Davis AP, Dolinski K, Dwight SS, Eppig JT, et al: Gene ontology: Tool for the unification of biology. The gene ontology consortium. Nat Genet 25: 25-29, 2000.

33. The Gene Ontology Resource: 20 years and still Going strong. Nucleic Acids Res 47: D330-D338, 2019.

34. Zhou Y, Zhou B, Pache L, Chang M, Khodabakhshi AH, Tanaseichuk O, Benner C and Chanda SK: Metascape provides a biologist-oriented resource for the analysis of systems-level datasets. Nat Commun 10: 1523, 2019.

35. Ding H, Gui X, Lin X, Chen R, Ma T, Sheng Y, Cai H and Fen Y: The prognostic effect of MAC30 expression on patients with non-small cell lung cancer receiving adjuvant chemotherapy. Technol Cancer Res Treat 16: 645-653, 2017. 
36. Wang $\mathrm{H}$, Zou L, Ma K, Yu J, Wu H, Wei M and Xiao Q Cell-Specific mechanisms of TMEM16A Ca ${ }^{2+}$-activated chloride channel in cancer. Mol Cancer 16: 152, 2017.

37. Guo J, Chen L, Luo N, Yang W, Qu X and Cheng Z: Inhibition of TMEM45A suppresses proliferation, induces cell cycle arrest and reduces cell invasion in human ovarian cancer cells. Oncol Rep 33: 3124-3130, 2015.

38. Zhao J, Zhu D, Zhang X, Zhang Y, Zhou J and Dong $\mathrm{M}$ : TMEM206 promotes the malignancy of colorectal cancer cells by interacting with AKT and extracellular signal-regulated kinase signaling pathways. J Cell Physiol 234: 10888-10898, 2019.

39. Ullrich F, Blin S, Lazarow K, Daubitz T, von Kries JP and Jentsch TJ: Identification of TMEM206 proteins as pore of PAORAC/ASOR acid-sensitive chloride channels. ELife 8: e49187, 2019.

40. Lin T, Zhou S, Gao H, Li Y and Sun L: MicroRNA-325 is a potential biomarker and tumor regulator in human bladder cancer. Technol Cancer Res Treat 17: 1533033818790536, 2018.
41. Zhang Z, Han Y, Sun G, Liu X, Jia X and Yu X: MicroRNA-325-3p inhibits cell proliferation and induces apoptosis in hepatitis $B$ virus-related hepatocellular carcinoma by down-regulation of aquaporin 5. Cell Mol Biol Lett 24: 13, 2019.

42. Yao S, Zhao T and Jin H: Expression of MicroRNA-325-3p and its potential functions by targeting HMGB1 in non-small cell lung cancer. Biomed Pharmacother 70: 72-79, 2015.

43. Chen D, Li Y, Yu Z, Li Y, Su Z, Ni L, Yang S, Gui Y and Lai Y: Downregulated microRNA-510-5p acts as a tumor suppressor in renal cell carcinoma. Mol Med Rep 12: 3061-3066, 2015.

(i) (9) This work is licensed under a Creative Commons Attribution-NonCommercial-NoDerivatives 4.0 International (CC BY-NC-ND 4.0) License. 